GISELE FARIA

\title{
Toxicidade da clorexidina injetada na pata de camundongos e adicionada em cultura de fibroblastos L929
}

Tese apresentada à Faculdade de Medicina de Ribeirão Preto da Universidade de São Paulo para obtenção do título de Doutor em Ciências Médicas. Área de Concentração: Patologia. Opção: Patologia Experimental

Orientador: Prof. Dr. Marcos A. Rossi

Ribeirão Preto

2007 
Faria, Gisele

Toxicidade da clorexidina injetada na pata de camundongos e adicionada em cultura de fibroblastos L929. Ribeirão Preto, 2007.

101 p. : il. ; $28 \mathrm{~cm}$

Tese de Doutorado, apresentada à Faculdade de Medicina de

Ribeirão Preto/USP. Área de concentração: Patologia, opção: Patologia Experimental.

Orientador: Rossi, Marcos Antonio.

1. Clorexidina. 2. Toxicidade. 3. Morte celular. 4. Estresse do retículo endoplasmático. 5. Fibroblastos L929. 6. Edema da pata.

7. Lesão periapical. 


\section{RESUMO}

Faria G. Toxicidade da clorexidina injetada na pata de camundongos e adicionada em cultura de fibroblastos L929 [tese]. Ribeirão Preto: Faculdade de Medicina de Ribeirão Preto da Universidade de São Paulo; 2007.

Como a clorexidina (CHX) tem sido recomendada como solução irrigadora de canais radiculares e como curativo de demora, o objetivo do presente estudo foi caracterizar in vivo a lesão induzida pela injeção de CHX a 0,125, 0,25, 0,5 e 1,0\% na pata de camundongos em intervalos de tempo selecionados ( 24 e 48 horas e 7 e 14 dias) e in vitro o modo e a causa morte celular (necrose e/ou apoptose) e o estresse causado pela exposição de fibroblastos L929 em cultura a concentrações crescentes da droga $(0,000125,0,00025,0,0005,0,001,0,002,0,004,0,008$ e 0,016\%) por 24 horas. A proliferação celular foi avaliada por meio da incorporação de metil-3H-timidina ao DNA das células e imunomarcação para PCNA. A ultraestrutura foi analisada em microscópio eletrônico de transmissão e de varredura e o citoesqueleto das células por meio de marcação para actina e $\alpha$-tubulina. Citometria de fluxo (Anexina- $\mathrm{V}$ FITC/iodeto de propídeo) foi empregada para diferenciar células necróticas de apoptóticas. Também, foi efetuada marcação para retículo endoplasmático, Bcl-2 (B-célula CLL/linfoma 2), Hsp70 (proteína de choque térmico 70) e Grp78 (glucose-regulated protein 78). Quando injetada no espaço subplantar da pata traseira de camundongos, a CHX induziu alterações necróticas na epiderme, derme e tecido subcutâneo em associação com uma resposta inflamatória reacional, particularmente nas concentrações mais elevadas. Em cultura de fibroblastos, a CHX induziu a diminuição da proliferação celular, causou morte celular por apoptose e necrose, desestruturação do citoesqueleto, alteração da morfologia celular, aumento da área das células, dilatação do retículo endoplasmático rugoso e acúmulo de proteínas nas cisternas. Além disso, a CHX causou aumento da expressão de Hsp70, de Grp78 (indicadores de estresse celular) e de Bcl-2 (proteína anti-apoptótica). Em conclusão, a CHX injetada no espaço subplantar da pata traseira de camundongos induz efeitos tóxicos severos. Além disso, a CHX adicionada em cultura de fibroblastos causou estresse do retículo endoplasmático como consequência do acúmulo de proteínas nas cisternas e induziu a morte celular por necrose e apoptose via estresse do retículo endoplasmático, além de causar estresse celular. Os resultados sugerem que a CHX poderia ter um efeito desfavorável na resolução de lesões periapicais em decorrência de sua ação tóxica sobre as células do tecido em torno do ápice dentário.

Palavras chave: Clorexidina, toxicidade, morte celular, estresse do retículo endoplasmático, fibroblastos L929, edema da pata, lesão periapical. 


\section{ABSTRACT}

Faria G. Chlorhexidine toxicity injected in the paw of mice and added to cultured L929 fibroblasts [tesis]. Ribeirão Preto: Faculty of Medicine of Ribeirão Preto, University of São Paulo; 2007.

Because chlorhexidine (CHX) has been recommended as either endodontic irrigant or root canal dressing, this study aimed to characterize in vivo the lesion induced by injection of $\mathrm{CHX}$ at concentrations of $0.125,0.25,0.5$ and $1.0 \%$ in the paw of mice at selected time intervals (24 and 48 hours and 7 and 14 days) and in vitro the mode and the cause of cell death (necrosis and /or apoptosis), and the cellular stress caused by exposition of cultured L929 fibroblasts to ascending concentrations of the drug $(0.000125,0.00025,0.0005,0.001,0.002,0.004,0.008$ and $0.016 \%$ ) for 24 hours. The cell proliferation assay was performed by measuring incorporation of metil-3H-thymidine to cell DNA and immunocytochemical staining analysis of PCNA. The cell ultrastructure was analyzed in transmission and scanning electron microscopes and the cell cytoskeleton by florescence analysis of actin and $\alpha$ tubulin. Apoptosis and necrosis were discriminated by flow cytometry with annexin V-FITC and PI. In addition endoplasmic reticulum, Bcl-2 (B-cell CLL/lymphoma 2), Hsp70 (heat shock protein 70) and Grp78 (glucoseregulated protein 78) were detected by fluorescence. CHX injected in the sub plantar space of the hind paw of mice induced necrotic changes in the epidermis, dermis and subcutaneous tissue in association with reactive inflammatory response, particularly at higher concentrations. In cultured fibroblasts, CHX decreased cellular proliferation, caused cell death by apoptosis and necrosis, disruption of the cytoskeleton, morphological cellular alterations, increased cells size (area), rough endoplasmic reticulum dilatation with accumulation of cysternal protein. In addition, CHX induced increased expression of Hsp 70, Grp78 (indicators of cellular stress) and Bcl-2 (anti-apoptotic protein). It was concluded that CHX injected in the sub plantar space of the hind paw of mice could induce severe toxic effect. In addition CHX caused endoplasmic reticulum stress as a consequence of accumulation of proteins in the endoplasmic reticulum cysterns and induced cell death by apoptosis and necrosis via endoplasmic reticulum stress and caused cellular stress. Taken together, these findings suggest that CHX may have an unfavorable effect on the resolution of apical periodontitis, due a toxic effect on the periapical tissue cells.

Key words: Chlorhexidine, toxicity, cell death, endoplasmic reticulum stress, L929 fibroblasts, paw edema, apical periodontitis. 


\section{LISTA DE ABREVIATURAS E SIGLAS}

Bcl-2: (B-cell CLL/lymphoma 2)

FACS: separador celular ativado por fluorescência

FITC: isotiocianato de fluoresceína

Grp78: glucose regulated protein

Hsp70: proteína de choque térmico 70 (heat shock protein 70)

PCNA: antígeno nuclear de proliferação celular

PI: iodeto de propídeo

RE: retículo endoplasmático

DMSO: Dimetilsulfóxido

BSA: albumina de soro bovino

DAB: diaminobenzidina

DAPI: 4’,6-diamidino-2-fenilindol 


\section{SUMÁRIO}

1 INTRODUÇÃO 14

2 MATERIAIS E MÉTODOS 17

$\begin{array}{lll}2.1 & \text { Estudo in vivo } & 17\end{array}$

$\begin{array}{lll}2.2 & \text { Estudo in vitro } & 20\end{array}$

3 RESULTADOS 28

3.1 Estudo in vivo 28

3.2 Estudo in vitro 31

4 DISCUSSÃo $\quad 67$

5 CONCLUSÃO

REFERÊNCIAS

$\begin{array}{lr}\text { ANEXOS } & 84\end{array}$ 


\section{INTRODUÇÃO}

O sucesso do tratamento endodôntico de dentes com necrose pulpar e lesão periapical depende de muitos fatores, sendo que o mais importante é a redução ou eliminação da infecção bacteriana (Tronstad, 1992). O preparo biomecânico, empregando instrumentos manuais ou rotatórios, associado a soluções irrigadoras é uma fase do tratamento endodôntico que visa, dentre outras finalidades, o combate à infecção (Leonardo, 2005). Entretanto, como as bactérias não se encontram localizadas somente na luz do canal radicular principal (Leonardo et al., 2002; Leonardo et al., 1994, Leonardo \& Silva, 2005), o preparo biomecânico, isoladamente, não é eficaz na sua eliminação (Rodrigues \& Biffi, 1989; Leonardo et al., 1994; Faria et al., 2005). Durante o tratamento endodôntico, devem ser empregadas substâncias que irão combater não somente a infecção remanescente na luz e paredes do canal radicular, mas principalmente aquela situada profunda e difusamente pela estrutura do dente, áreas estas inacessíveis ao preparo biomecânico e ao sistema de defesa do organismo (Dalton et al., 1998; Shuping et al., 2000; Faria et al., 2005; Leonardo et al., 2006). Com esse objetivo, diferentes substâncias têm sido utilizadas como curativo de demora em canais radiculares de dentes com necrose pulpar e lesão periapical (Barbosa et al., 1997)

Um curativo de demora ideal deve apresentar eficácia antimicrobiana e não causar danos aos tecidos periapicais. O hidróxido de cálcio $\left[\mathrm{Ca}(\mathrm{OH})_{2}\right]$ apresenta esses requisitos e atualmente é considerado a medicação intracanal de escolha (Carrote et al., 2004). No entanto, tem sido demonstrado que o Enterococcus faecalis apresenta resistência ao $\mathrm{Ca}(\mathrm{OH})_{2}($ Ercan et al., 2006) e a falha de muitos tratamentos endodônticos tem sido atribuída a esse fato (Williams et al., 2006).

Recentemente, a clorexidina (CHX) em diferentes concentrações tem sido recomendada como curativo de demora (Basrani et al., 2002; Leonardo \& Silva, 2005; Oncaag et al., 2006; Manzur et al., 2007). Quando empregada para essa finalidade, a CHX mostra ser mais efetiva do que o $\mathrm{Ca}(\mathrm{OH})_{2}$ na eliminação dos Enterococcus faecalis presentes nos túbulos dentinários (Heling et al., 1992). Com a finalidade de obter um maior efeito antibacteriano, tem sido proposta a utilização da pasta de $\mathrm{Ca}(\mathrm{OH})_{2}$ em combinação com a $\mathrm{CHX}$ como curativo de demora (Soares et al., 2006; Oncaag et al., 2006; Manzur et al., 2007). No entanto, estudo recente em nosso laboratório mostrou que a adição de $\mathrm{CHX}$ a $1,0 \%$ à pasta de $\mathrm{Ca}(\mathrm{OH})_{2}$, como medicação 
intracanal, retardou o processo de reparo de lesões periapicais de dentes de cães induzidas experimentalmente (De Rossi et al., 2005).

A CHX é uma biguanida descoberta na década de 40 por pesquisadores que buscavam desenvolver agentes antimalária (Parson, 1974). Ela é uma base forte, praticamente insolúvel em água, que reage com ácidos, formando sais. Os sais originais eram o acetato e o cloridrato de CHX, ambos com baixa solubilidade em água. Por isso eles foram substituídos pelo digluconato de CHX. Esse sal não pode ser isolado na forma sólida e por isso é manufaturado como solução aquosa a 20\% (Foulkes, 1973; Denton, 1991). A molécula da CHX consiste de dois anéis de 4clorofenol e dois grupos bisbiguanidas, simetricamente ligados a uma cadeia hexametileno, fornecendo propriedades hidrófilas e hidrófobas (Thylstrup \& Fejerskov, 2001). A CHX apresenta amplo espectro de ação contra bactérias gram-positivas e gram-negativas, e se liga aos tecidos dentais e às membranas mucosas apresentando liberação prolongada em níveis terapêuticos. Sua atividade antimicrobiana origina-se de sua carga positiva em $\mathrm{pH}$ fisiológico, facilitando sua adesão de forma não específica à parede bacteriana, carregada negativamente. Essa interação ocasiona uma alteração no equilíbrio osmótico bacteriano, levando ao extravasamento de potássio e fósforo e precipitação do citoplasma, com consequente morte da bactéria (Hidalgo \& Dominguez, 2001). Esse agente antimicrobiano tem sido amplamente utilizado na área médica para antissepsia de queimaduras, de feridas cirúrgicas, da pele e de mucosas (Hidalgo \& Dominguez, 2001). Em Odontologia, a CHX é muito empregada nas terapias periodontal (Cosyn et al., 2006; Faveri et al., 2006) e endodôntica (Leonardo et al., 1999; Yamashita et al., 2003; Portenier et al., 2005; Dunavant et al., 2006; Oncaag et al., 2006; Zehnder, 2006; Regan \& Fleury, 2006; Manzur et al., 2007), para a prevenção da cárie dental (Du et al., 2006), na antissepsia de feridas cirúrgicas (Caso et al., 2005; Metin et al., 2006), entre outros propósitos (Denton, 1991).

Há na literatura evidências conflitantes sobre a toxicidade da CHX. Apesar de estudos terem mostrado que a CHX apresenta efeito benéfico (Sanches et al., 1988; Heitz et al., 2004) não afetando a síntese de colágeno (Brennan et al.,1986) e não interferindo no processo de reparo de feridas cirúrgicas (Hirst et al.,1973), outros afirmam que essa droga apresenta um efeito claro no retardo da formação do tecido de granulação (Paunio et al., 1978), induzindo alterações microcirculatórias (Luostarinen et al., 1977) e retardamento do reparo tecidual (Bassetti \& Kallenberger, 1980; Saatman et al., 1986). Algumas pesquisas têm mostrado que a CHX nas 
concentrações de $0,12,1,0$ e 2,0\% induz reação inflamatória quando injetada no tecido subcutâneo, apesar da causa dessa reação não ter sido relatada (Yesilsoy et al., 1995; Delgado, 2002; Önçăg et al., 2003).

Efeitos citotóxicos da CHX podem explicar o retardo no processo de reparo tecidual. Estudos in vitro têm mostrado que a $\mathrm{CHX}$ apresenta efeitos tóxicos em uma variedade de células eucarióticas, sendo que um dos prováveis mecanismos de toxicidade está relacionado à superfície eletrostática (Hidalgo \& Dominguez, 2001). A CHX causa danos à membrana de células eucarióticas levando a um aumento da permeabilidade ao cálcio, acompanhado pela liberação de lactato desidrogenase e citólise (Knuutilla \& Soderling, 1981; Glaber et al., 1987; Babich et al., 1995). No entanto, o aumento da permeabilidade celular devido à alta afinidade da CHX por radicais orgânicos carregados negativamente não parece ser o único mecanismo de toxicidade. A CHX inibe a síntese de DNA (Hidalgo \& Dominguez, 2001), a síntese de proteína (Goldschmidt et al., 1977; Purcher \& Daniel, 1992; Mariotti \& Rumph, 1999; Chang et al.; 2001), a atividade mitocondrial (Chang et al., 2001; Hidalgo \& Dominguez, 2001) e a proliferação celular (Purcher \& Daniel, 1992; Mariotti and Rumpf, 1999; Hidalgo \& Dominguez, 2001). Além disso, foi demonstrado que a CHX é citotóxica em cultura de fibroblastos de humanos obtidos de biópsias de pele em concentrações $\geq 0,0025 \%$ por 24 horas, causando morte de todas as células devido à diminuição de ATP (Hidalgo \& Dominguez, 2001). No entanto, o mecanismo intrínseco sob o qual a CHX induz à citotoxicidade não está claramente compreendido.

O objetivo do presente trabalho foi caracterizar in vivo a lesão induzida pela injeção de várias concentrações de CHX no espaço subplantar da pata traseira de camundongos, e, in vitro, a causa e o modo de morte celular (necrose e/ou apoptose), além do estresse celular produzido pela exposição de fibroblastos L929 em cultura a concentrações crescentes da droga. 


\section{REFERÊNCIAS}

Babich H, Wurzburger BJ, Rubin YL, Sinensky MC, Blau L. An in vitro stydy on the cytotoxicity of chlorhexidine digluconate to human gingival cells. Cell Biol and Toxicol 1995;11:79-88.

Barbosa CA, Goncalves RB, Siqueira JF Jr, De Uzeda M. Evaluation of the antibacterial activities of calcium hydroxide, chlorhexidine, and camphorated paramonochlorophenol as intracanal medicament. A clinical and laboratory study. J Endod 1997;23:297-300.

Basrani B, Santos JM, Tjäderhane L, Grad H, Gorduysus O, Huang J, Lawrence HP, Friedman S. Substantive antimicrobial activity in chlorhexidine-treated human root dentin. Oral Surg Oral Med Oral Pathol Oral Radiol Endod 2002;94:240-5.

Bassetti C, Kallenberger A. Influence of chlorhexidine rinsing on the healing of oral mucosa and osseous lesions. J Clin Periodontol 1980;7:443-56.

Bedard K, MacDonald N, Collins J, Cribb A. Cytoprotection following endoplasmic reticulum stress protein induction in continuous cell lines. Basic Clin Pharmacol Toxicol 2004;94:124-31.

Brennan SS, Foster ME, Leaper DJ. Antiseptic toxicity in wounds healing by secondary intention. J Hosp Infect 1986;8:263-7.

Carrotte P. Endodontics: Part 9. Calcium hydroxide, root resorption, endo-perio lesions. Br Dent J 2004;197:735-43.

Caso A, Hung LK, Beirne OR. Prevention of alveolar osteitis with chlorhexidine: a meta-analytic review. Oral Surg Oral Med Oral Pathol Oral Radiol Endod 2005;99:155-9.

Chang YC, Huang FM, Tai KW, Chou MY. The effect of sodium hypochlorite and chlorhexidine on cultured human periodontal ligament cells. Oral Surg Oral Med Oral Pathol Oral Radiol Endod 2001;92:446-50.

Ciapetti G, Granchi D, Cenni E, Savarino L, Cavedagna D, Pizzoferrato A. Cytotoxic effect of bone cements in HL-60 cells: distinction between apoptosis and necrosis. J Biomed Mater Res 2000;52:338-45.

Cosyn J, Wyn I, De Rouck T, Moradi Sabzevar M. Clinical benefits of subgingival chlorhexidine varnish application as an adjunct to same-day full-mouth root planing: a pilot study. J Periodontol 2006;77:1074-9.

Cribb AE, Peyrou M, Muruganandan S, Schneider L. The endoplasmic reticulum in xenobiotic toxicity. Drug Metab Rev 2005;37:405-42.

Dabrowska MI, Becks LL, Lelli JL Jr, Levee MG, Hinshaw DB. Sulfur mustard induces apoptosis and necrosis in endothelial cells. Toxicol Appl Pharmacol 1996;141:568-83. 
Dalton BC, Orstavik D, Phillips C, Pettiette M, Trope M. Bacterial reduction with nickel-titanium rotary instrumentation. J Endod 1998;24:763-7.

De Rossi A, Silva LA, Leonardo MR, Rocha LB, Rossi MA. Effect of rotary or manual instrumentation, with or without a calcium hydroxide/1\% chlorhexidine intracanal dressing, on the healing of experimentally induced chronic periapical lesions. Oral Surg Oral Med Oral Pathol Oral Radiol Endod 2005;99:628-36.

Delgado EMS. Estudo da biocompatibilidade de uma pasta à base de hidróxido de cálcio associada à clorexidina a $1 \%$, no tecido conjuntivo subcutâneo da pata de ratos [dissertação]. Araraquara: Faculdade de Odontologia, Universidade Estadual Paulista; 2002.

Denton GW. Chlorhexidine. In: Block SS, editors. Disinfection, sterilization and preservations. $4^{\text {th }}$ ed. Philadelphia: Ed. Lea \& Febiger; 1991. p.274-89.

DesMarais V, Ghosh M, Eddy R, Condeelis J. Cofilin takes the lead. J Cell Sci 2005;118:19-26.

Domnina LV, Ivanova OY, Cherniak BV, Skulachev VP, Vasiliev JM. Effects of the inhibitors of dynamics of cytoskeletal structures on the development of apoptosis induced by the tumor necrosis factor. Biochemistry (Mosc) 2002;67:737-46.

Du MQ, Tai BJ, Jiang H, Lo EC, Fan MW, Bian Z. A two-year randomized clinical trial of chlorhexidine varnish on dental caries in Chinese preschool children. J Dent Res 2006;85:557-9.

Dunavant TR, Regan JD, Glickman GN, Solomon ES, Honeyman AL. Comparative evaluation of endodontic irrigants against Enterococcus faecalis biofilms. J Endod 2006;32:527-31.

Ercan E, Dalli M, Dülgergil CT. In vitro assessment of the effectiveness of chlorhexidine gel and calcium hydroxide paste with chlorhexidine against Enterococcus faecalis and Candida albicans. Oral Surg Oral Med Oral Pathol Oral Radiol Endod 2006;102:e27-e31.

Eriksson JE, Paatero GIL, Merilueto JAO, Codd GA, Kass GEN, Nicotera P, Orrenius S. Rapid microfilament reorganization induced in isolated rat hepatocytes by microcystin-LR, a cyclic peptide toxin. Exp Cell Res 1989;185:86-100.

Euteneuer U, Schliwa M. Persistent, directional motility of cells and cytoplasmic fragments in the absence of microtubules. Nature 1984;310:58-61.

Faria G, Nelson-Filho P, Freitas AC, Assed S, Ito IY. Antibacterial effect of root canal preparation and calcium hydroxide paste (Calen) intracanal dressing in primary teeth with apical periodontitis. J Appl Oral Sci 2005;13:351-5.

Faveri M, Gursky LC, Feres M, Shibli JA, Salvador SL, de Figueiredo LC. Scaling and root planing and chlorhexidine mouthrinses in the treatment of chronic periodontitis: a randomized, placebo-controlled clinical trial. J Clin Periodontol 2006;33:819-28.

Foulkes DM. Some toxicological observations on chlorhexidine. J Periodontal Res Suppl $1973 ; 12: 55-60$. 
Gabler WL, Bullock WW, Creamer HR. The influence of chlorhexidine on superoxide generation by induced human neutrophils. J Periodontal Res 1987;22:445-50.

Goldschmidt P, Cogen R, Taubman S. Cytopathologic effects of chlorhexidine on human cells.

Gunaratnam M, Grant MH. Damage to F-actin and cell death induced by chromium VI and nickel in primary monolayer cultures of rat hepatocytes. Toxicol In Vitro 2004;18:245-53.

Harding HP, Calfon M, Urano F, Novoa I, Ron D. Transcriptional and translational control in the Mammalian unfolded protein response. Annu Rev Cell Dev Biol 2002;18:575-99.

Heitz F, Heitz-Mayfield LJ, Lang NP. Effects of post-surgical cleansing protocols on early plaque control in periodontal and/or periimplant wound healing. $J$ Clin Periodontol 2004;31:1012-8.

Heling I, Steinberg D, Kenig S, Gavrilovich I, Sela MN, Friedman M. Efficacy of a sustained release device containing chlorhexidine and $\mathrm{Ca}(\mathrm{OH})_{2}$ in preventing secondary infection of dentinal tubules. Int Endod J 1992;25:20-4.

Hernandez EP, Botero TM, Mantellini MG, McDonald NJ, Nör JE. Effect of Proroot MTA mixed with chlorhexidine on apoptosis and cell cycle of fibroblasts and macrophages in vitro. Int Endod J 2005;38:137-43.

Hidalgo E, Domingues C. Mechanisms underlying chlorhexidine-induced cytotoxicity. Toxicol In Vitro 2001;15:271-6.

Hightower LE. Heat shock, stress proteins, chaperones, and proteotoxicity. Cell 1991;66:191-7.

Hirst RC, Egelberg R, Hornbuckle GC, Oliver RC, Rothbun WE. Microscopic evaluation of topically applied chlorhexidine gluconate on gingival wound healing in dogs. J Southern Calif State Dent Assoc 1973;41:311-17.

Hooser SB, Beasley VR, Waite LL, Kuhlenschmidt MS, Carmichael WW, Haschek WM. Actinfilament alterations in rat hepatocytes induced in vivo and in vitro by microcystin-LR, a hepatotoxin from the blue-green alga Microcystis aeruginosa. Vet Pathol 1991;28:259-266.

Inagi R, Nangaku M, Onogi H, Ueyama H, Kitao Y, Nakazato K, Ogawa S, Kurokawa K, Couser WG, Miyata T. Involvement of endoplasmic reticulum (ER) stress in podocyte injury induced by excessive protein accumulation. Kidney Int 2005;68:2639-50.

International Organization for Standardization (ISO). ISO 10993-5: biological evaluation of medical devices - Part 5: tests for in vitro cytotoxicity. Switzerland; 1999.

J Periodontol 1977;48:212-5.

Jarosch E, Lenk U, Sommer T. Endoplasmic reticulum-associated protein degradation. Int Rev Cytol 2003;223:39-81. 
Johnson HJ, Northup SJ, Seagraves PA, Garvin PJ, Wallin RF. Biocompatibility test procedures for materials evaluation in vitro. I. Comparative test system sensitivity. J Biomed Mater Res $1983 ; 17: 571-86$.

Khan SA, Ghosh S, Wickstrom M, Miller LA, Hess R, Haschek W, Beasley VR. Comparative pathology of microcystin-LR in cultured hepatocytes, fibroblasts, and renal epithelial cells. Natural Toxins 1995;3:119-128.

Kluck RM, Bossy-Wetzel E, Green DR, Newmeyer DD. The release of cytochrome c from mitochondria: a primary site for Bcl-2 regulation of apoptosis. Science 1997;275:1132-36.

Knuuttila J, Söderling E. Effect of chlorhexidine on the release of lysosomal enzymes from cultured macrophages. Acta Odontol Scand 1981;39:285-9.

Kopnin BP. Targets of oncogenes and tumor suppressors: key for understanding basic mechanisms of carcinogenesis. Biochemistry (Mosc) 2000;65:2-27.

Kroemer G. Mitochondrial control of apoptosis: an introduction. Biochem Biophys Res Commun 2003;304:433-5.

Kumar V, Abbas AK, Fausto N. Cell adaptations, cell injury, and cell death. In: Robbins and Cotran. Pathologic basis of disease. $7^{\text {th }}$ ed. Philadelphia: Elsevier Saunders; 2005. p.3-46.

Lee AS. The ER chaperone and signaling regulator GRP78/BiP as a monitor of endoplasmic reticulum stress. Methods 2005;35:373-81.

Leonardo MR, Almeida WA, Ito IY, da Silva LA. Radiographic and microbiologic evaluation of posttreatment apical and periapical repair of root canals of dogs' teeth with experimentally induced chronic lesion. Oral Surg Oral Med Oral Pathol 1994;78:232-8.

Leonardo MR, Hernandez ME, Silva LA, Tanomaru-Filho M. Effect of a calcium hydroxidebased root canal dressing on periapical repair in dogs: a histological study. Oral Surg Oral Med Oral Pathol Oral Radiol Endod 2006;102:680-5.

Leonardo MR, Rossi MA, Silva LA, Ito IY, Bonifacio KC. EM evaluation of bacterial biofilm and microorganisms on the apical external root surface of human teeth. J Endod 2002;28:815-8.

Leonardo MR, Silva LAB. Filosofia do tratamento de canais radiculares. Necropulpectomia: conceituação. In: Leonardo MR. Endodontia: tratamento de canais radiculares: princípios técnicos e biológicos. São Paulo: Artes Médicas; 2005. p.123-66.

Leonardo MR, Tanomaru Filho M, Silva LA, Nelson Filho $\mathrm{P}$, Bonifacio KC, Ito IY. In vivo antimicrobial activity of $2 \%$ chlorhexidine used as a root canal irrigating solution. J Endod 1999;25:167-71.

Leonardo MR. Endodontia: tratamento de canais radiculares: princípios técnicos e biológicos. São Paulo: Artes Médicas; 2005. cap.13, p.487-540. 
Li X, Liu Y, Song L. Cytological alterations in isolated hepatocytes from common carp (Cyprinus carpio L.) exposed to microcystin-LR. Environ Toxicol 2001;16:517-22.

Lima C, Bianca Clissa P, Piran-Soares A, Tanjoni I, Moura-da-Silva AM, Lopes-Ferreira M. Characterisation of local inflammatory response induced by Thalassophryne nattereri fish venom in a mouse model of tissue injury. Toxicon 2003;42:499-507.

Liu-Snyder P, McNally H, Shi R, Borgens RB. Acrolein-mediated mechanisms of neuronal death. J Neurosci Res 2006;84:209-18.

Lowe DM, Moore MN, Readman JW. Pathological reactions and recovery of hepatopancreatic digestive cells from the marine snail Littorina littorea following exposure to a polycyclic aromatic hydrocarbon. Mar Environ Res 2006;61:457-70.

Luostarinen V, Soderling E, Knuuttila M, Paunio K. Effect of chlorhexidine on the hamster cheek pouch. Microcirculation and penetration studies. J Periodontol 1977;48:421-4.

Majno G, Joris I. Cells, tissues, and disease: principles of general pathology. $2^{\text {nd }}$ ed. Oxford University Press: New York; 2004. cap.4, p.128-85.

Mannherz HG, Gonsior SM, Wu X, Polzar B, Pope BJ, Wartosch L, Weeds AG. Dual effects of staurosporine on A431 and NRK cells: microfilament disassembly and uncoordinated lamellipodial activity followed by cell death. Eur J Cell Biol 2006;85:785-802.

Mantellini MG, Botero TM, Yaman P, Dennison JB, Hanks CT, Nor JE. Adhesive resin induces apoptosis and cell-cycle arrest of pulp cells. J Dent Res 2003;82:592-6.

Manzur A, Gonzalez AM, Pozos A, Silva-Herzog D, Friedman S. Bacterial quantification in teeth with apical periodontitis related to instrumentation and different intracanal medications: a randomized clinical trial. J Endod 2007;33:114-8.

Mariotti AJ, Rumpf DA. Chlorhexidine-induced changes to human gingival fibroblast collagen and non-collagen protein production. J Periodontol 1999;70:1443-8.

Metcalfe A, Streuli C. Epithelial apoptosis. Bioessays 1997;19:711-20.

Metin M, Tek M, Sener I. Comparison of two chlorhexidine rinse protocols on the incidence of alveolar osteitis following the surgical removal of impacted third molars. J Contemp Dent Pract 2006;779-86.

Neumann A, Reske T, Held M, Jahnke K, Ragoss C, Maier HR. Comparative investigation of the biocompatibility of various silicon nitride ceramic qualities in vitro. J Mater Sci Mater Med 2004; 15:1135-40.

Newton APN, Cadena SMSC, Rocha MEM, Carnieri EGS, Oliveira MBM. New data on biological effects of chlorhexidine: $\mathrm{Fe} 2+$ induced lipid peroxidation and mitochondrial permeability transition. Toxicol Lett 2004;151:407-16. 
Oncaag O, Gogulu D, Uzel A. Efficacy of various intracanal medicaments against Enterococcus faecalis in primary teeth: an in vivo study. J Clin Pediatr Dent 2006;30:233-7.

Önçağ Ö, Hoşgör M, Hilmioğlu S, Zekioğlu O, Eronat C, Burhanoğlu D. Comparison of antibacterial and toxic effects of various root canal irrigants. Int Endod J 2003;36: 423-32.

Ou J, Ou Z, McCarver DG, Hines RN, Oldham KT, Ackerman AW, Pritchard KAJr. Trichloroethylene decreases heat shock protein 90 interactions with endothelial nitric oxide synthase: implications for endothelial cell proliferation. Toxicol Sci 2003;73:90-7.

Oyadomari S, Araki E, Mori M. Endoplasmic reticulum stress-mediated apoptosis in pancreatic $\beta$-cells. Apoptosis 2002;7:335-45.

Parsell DA, Lindquist S. The function of heat-shock proteins in stress tolerance: degradation and reactivation of damaged proteins. Annu Rev Genet 1993;27:437-96.

Parson JC. Chemotherapy of dental plaque: a review. J Periodontol 1974;45:177-86.

Paunio KU, Knuttila M, Mielitynen $\mathrm{H}$. The effect of chlorhexidine gluconate on the formation of experimental granulation tissue. J Periodontol 1978;49:92-5.

Pollard TD, Borisy GG. Cellular motility driven by assembly and disassembly of actin filaments. Cell 2003; 112:453-65. Erratum in: Cell. 2003;113:549.

Portenier I, Waltimo T, Orstavik D, Haapasalo M. The susceptibility of starved, stationary phase, and growing cells of Enterococcus faecalis to endodontic medicaments. J Endod 2005;31:380-6.

Pucher JJ, Daniel JC. The effects of chlorhexidine digluconate on human fibroblasts in vitro. J Periodontol 1992;63:526-32.

Reed JC. Bcl-2 family proteins: strategies for overcoming chemoresistance in cancer. Adv Pharmacol 1997a;41:501-32.

Reed JC. Double identity for proteins of the Bcl-2 family. Nature 1997b; 87:773-6.

Regan JD, Fleury AA. Irrigants in non-surgical endodontic treatment. J Ir Dent Assoc 2006;52:84-92.

Rodrigues HH, Biffi JC. A histobacteriological assessment of nonvital teeth after ultrasonic root canal instrumentation. Endod Dent Traumatol 1989;5:182-7.

Rudolf E, Cervinka M, Cerman J, Schroterova L. Hexavalent chromium disrupts the actin cytoskeleton and induces mitochondria-dependent apoptosis in human dermal fibroblasts. Toxicol In Vitro 2005;19:713-23.

Runnegar MT, Falconer IR. Effect of toxin from the cyanobacterium Microcystis aeruginosa on ultrastructural morphology and actin polymerization in isolated hepatocytes. Toxicon 1986;24:109-15. 
Saatman RA, Carlton WW, Hubben K, Spencer Streett C, Tuckosh JR, Debaecke PJ. A wound healing study of chlorhexidine digluconate in guinea pigs. Fund Appl Toxicol 1986;6:1-6.

Scanlon M, Laster SM, Wood JG, Gooding LR. Cytolysis by tumor necrosis factor is preceded by a rapid and specific dissolution of microfilaments. Proc Natl Acad Sci U S A. 1989;86:182-6.

Schmalz G. Use of cell cultures for toxicity testing of dental materials: advantages and limitations. J Dent 1994;22 Suppl 2:S6-11.

Schroder M, Kaufman RJ. The mammalian unfolded protein response. Annu Rev Biochem 2005;74:739-89.

Shuping GB, Orstavik D, Sigurdsson A, Trope M. Reduction of intracanal bacteria using nickeltitanium rotary instrumentation and various medications. J Endod 2000; 26:751-5.

Skulachev VP. The programmed death phenomena, aging, and the Samurai law of biology. Exp Gerontol 2001;36:995-1024.

Soares JA, Leonardo MR, Silva LAB, Tanomaru Filho M, Ito IY. Effect of rotary instrumentation and of the association of calcium hydroxide and chlorhexidine on the antisepsis of the root canal system in dogs. Braz Oral Res 2006;20:120-6.

Spångberg L, Engstrom B, Langeland K. Biologic effects of dental materials. 3. Toxicity and antimicrobial effect of endodontic antiseptics in vitro. Oral Surg Oral Med Oral Pathol 1973;36:856-71.

Tanomaru Filho M, Leonardo MR, da Silva LA. Effect of irrigating solution and calcium hydroxide root canal dressing on the repair of apical and periapical tissues of teeth with periapical lesion. J Endod 2002;28:295-9.

Thylstrup A, Fejerskov O. Cariologia Clínica. 2a ed. São Paulo: Ed. Santos; 2001.

Tronstad L. Recent development in endodontic research. Scand J Dent Res 1992;100:52-9.

Trump BF, Berezesky IK, Chang SH, Phelps PC. The pathways of cell death: oncosis, apoptosis, and necrosis. Toxicol Pathol 1997;25:82-8.

van de Water B, Kruidering M, Nagelkerke JF. F-actin disorganization in apoptotic cell death of cultured rat renal proximal tubular cells. Am J Physiol 1996;270:F593-F603.

Vermes I, Haanen C, Steffens-Nakken H, Reutelingsperger C. A novel assay for apoptosis. Flow cytometric detection of phosphatidylserine expression on early apoptotic cells using fluorescein labelled Annexin V. J Immunol Methods 1995;184:39-51.

Wallin RF, Arscott EF. A practical guide to ISO 10993-5: Cytotoxicity. MD\&DI April Column; 1998. Disponível em: http://www.devicelink.com/mddi/archive/98/04/013.html. 
Wilken R, Botha SJ, Grobler A, Germishuys PJ. In vitro cytotoxicity of chlorhexidine gluconate, benzydamine- $\mathrm{HCl}$ and povidone iodine mouthrinses on human gingival fibroblasts. SADJ 2001;56:455-60.

Williams JM, Trope M, Caplan DJ, Shugars DC. Detection and quantitation of E. faecalis by real-time PCR (qPCR), reverse transcription-PCR (RT-PCR), and cultivation during endodontic treatment. J Endod 2006;32:715-21.

Yamashita JC, Tanomaru Filho M, Leonardo MR, Rossi MA, Silva LA. Scanning electron microscopic study of the cleaning ability of chlorhexidine as a root-canal irrigant. Int Endod $\mathrm{J}$ 2003;36:391-4.

Yang J, Liu X, Bhalla K, Kim CN, Ibrado AM, Cai J, Peng TI, Jones DP, Wang X. Prevention of apoptosis by Bcl-2: release of cytochrome c from mitochondria blocked. Science 1997;275:112932.

Yenari MA, Giffard RG, Sapolsky RM. Steinberg GK. The neuroprotective potential of heat shock protein 70 (Hsp70). Mol Med Today 1999;5:525-31.

Yesilsoy C, Whitaker E, Cleveland D, Phillips E, Trope M. Antimicrobial and toxic effects of established and potential root canal irrigants. J Endod 1995;21:513-5.

Yujiri T, Fanger GR, Garrington TP, Schlesinger TK, Gibson S, Johnson GL. MEK kinase 1 (MEKK1) transduces c-Jun NH2-terminal kinase activation in response to changes in the microtubule cytoskeleton. J Biol Chem 1999;274:12605-10.

Zehnder M. Root canal irrigants. J Endod 2006;32:389-98.

Zhao L, Ackerman SL. Endoplasmic reticulum stress in health and disease. Curr Opin Cell Biol 2006;18:444-52. 
Anexo A - Certificado de aprovação do projeto de pesquisa pela Comissão de Ética em Experimentação Animal.

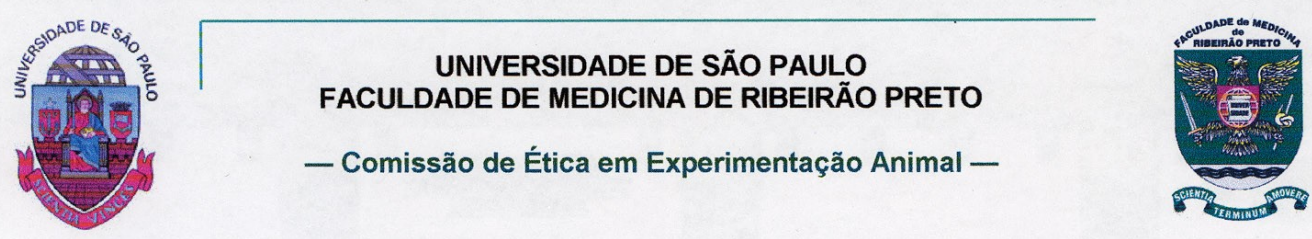

\section{E R T I F I C A D O}

Certificamos que o Protocolo para uso de animais em experimentação $\mathrm{n}^{\circ}$ 001/2006, sobre o projeto intitulado, "Estudo da biocompatibilidade de soluções de clorexidina no tecido conjuntivo subcutâneo da pata de camundongos", sob a responsabilidade do Prof. Dr. Marcos Antonio Rossi, está de acordo com os Princípios Éticos na Experimentação Animal adotado pelo Colégio Brasileiro de Experimentação Animal (COBEA) e foi APROVADO pela COMISSÃO DE ÉTICA EM EXPERIMENTAÇÃO ANIMAL (CETEA) em reunião de 27 de março de 2006.

(We certify that the protocol $\mathrm{n}^{\circ} 001 / 2006$, about "Biocompatibility evaluation of chlorhexidine solutions in subcutaneous connective tissue of mice paws", agrees with the ETHICAL PRINCIPLES IN ANIMAL RESEARCH adopted by Brazilian College of Animal Experimentation (COBEA) and was approved by the COLLEGE OF MEDICINE OF RIBEIRÃOO PRETO OF THE UNIVERSITY OF SÃO PAULO - ETHICAL COMMISSION OF ETHICS IN ANIMAL RESEARCH (CETEA) in 03/27/2006 meeting).

Ribeirão Preto, 28 de março de 2006.

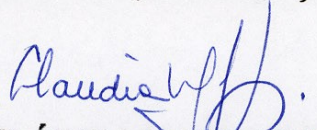

Profa. Dra. CLAUDIA MARIA LEITE MAFFEI

Vice-Presidente da Comissão

de Ética em Experimentação Animal 
Anexo B - Trabalhos resultantes dessa Tese até o presente momento.

Parte dos resultados desse trabalho foi aceita recentemente para publicação em uma revista de grande impacto na área de Odontologia: Gisele Faria, Mara R. N. Celes, Andiara De Rossi, Lea Assed B. Silva, João S. Silva, Marcos A. Rossi. Evaluation of Chlorhexidine Toxicity Injected in the Paw of Mice and Added to Cultured L929 Fibroblasts. Journal of Endodontics.

Obs: O trabalho foi enviado em 22 de novembro de 2006, retornou em 13 de dezembro de 2006 para pequenas alterações, foi aceito em 24 de dezembro de 2006 e a prova gráfica para correção nos foi enviada em 26 de janeiro de 2007.

Além disso, o trabalho foi apresentado na $23^{\text {a }}$ Reunião Anual da Sociedade Brasileira de Pesquisa Odontológica realizada no período de 4 a 6 de setembro de 2006 em Atibaia - SP , resultando em uma publicação: Gisele Faria; Mara Rúbia Nunes Celles, João Santana da Silva, Lea Assed Bezerra da Silva; Marcos Antonio Rossi. Clorexidina induz morte de fibroblastos por apoptose e, predominantemente por necrose. Brazilian Oral Reseach, v. 20, p. 158, 2006. 